\title{
Dr George Engelmann of St Louis (1847-1903) and the ethnology of childbirth
}

\author{
Peter M Dunn
}

George Julius Engelmann was born in St Louis, Missouri, USA, 2 July 1847. He obtained an $\mathrm{AB}$ at Washington University, St Louis, in 1867 (AM, 1870), but undertook most of his medical training in Europe, graduating as a doctor in Berlin in 1871 and as Master in Obstetrics in Vienna in 1872. After service in the Franco-Prussian war he returned to the USA to practise obstetrics in St Louis for two decades. There he became professor of obstetrics in the Missouri Medical College and St Louis Postgraduate School of Medicine, as well as consulting surgeon to the St Louis Female Hospital and physician in chief to the Maternity Hospital. He subsequently worked in Boston.

Engelmann's distinction in obstetrics was recognised at home and abroad. He was a founder member of the American Gynecological Society in 1876 and its president in 1900. He was also president of the Southern Surgical and Gynecological Society in 1890, and honorary president of the international congress in gynecology and obstetrics, 1892-1899. $\mathrm{He}$ was made an honorary member of many obstetric and scientific societies in both the USA and Europe.

While studying in Berlin in 1870-71, Engelmann came under the influence of the great Virchow who, among his many contributions, was the first medical man to develop the science of ethnology. Following in his footsteps Engelmann studied childbirth practices in the past as well as those still current among 'primitive' peoples. In 1882 he published his findings, ${ }^{1}$ writing in the preface: 'Why should ethnology not prove an aid in the development ... of medicine? A comparison of the crude methods of primitive peoples and peoples of former civilisations with the teachings of scientific obstetrics of today are amusing and interesting, but above all instructive and important.' The following extracts are taken from this book. ${ }^{1}$

Department of Child Health,

University of Bristol, Southmead Hospital, Southmead Road, Bristol BS10 5NB P M Dunn

Correspondence to: Professor P M Dunn.

\section{On the dorsal position in labour among civilised peoples:}

'The semi-recumbent position held sway in Europe ... until the last thirty or forty years, when it finally yielded completely to the dorsal decubitus, which is now almost universal among civilized people... Although

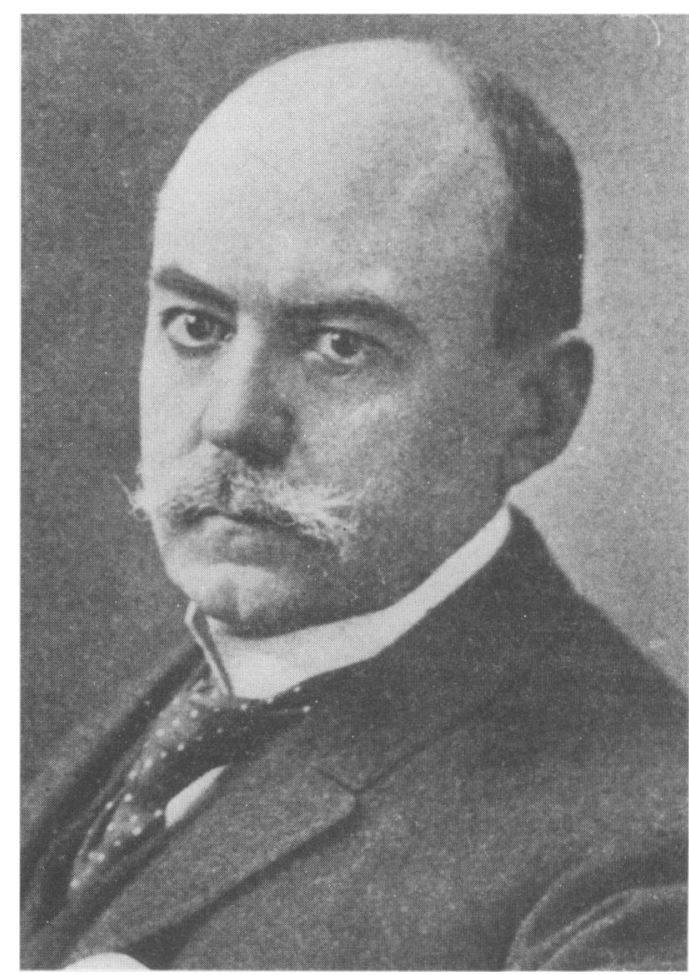

Professor George Englemann, 1847-1903.

dorsal decubitus is the position which is taught by the laws of modern obstetrics ... nature does not seem to have designed that woman should in this way free herself from her burden; at least it appears very strange that instinct, the correct guide of uncivilized people, should so rarely lead them to adopt the recumbent position...

It was not until I had ... begun to study the positions assumed ... during labor, that I began to understand that there was a method in the instinctive movements of women in the last stage of labor. I have seen them toss about, and sought to quiet them; I bade them have patience, and lie still on their backs; but, since entering upon this study, I have learned to look upon their movements in a very different light.

It has often appeared to me, as I sat watching a tedious labor case, how unnatural was the ordinary obstetric position for the parturient woman; the child is forced, I may say, upwards through the pelvic canal in the face of gravity, which acts in the intervals 
between the pains, and permits the presenting part of the child to sink back again, down the inclined canal. If we look upon the structure of the pelvis, more especially the direction of the pelvic canal and its axis, if we take into consideration the assistance which may be rendered by gravity, and, above all, by the abdominal muscles, the present obstetric position seems indeed a peculiar one. We know that the squatting position is the one naturally assumed if an effort is required to expel the contents of the pelvic viscera; we, moreover, all know how difficult, even impossible, it is for many to perform those functions recumbent in bed, and mainly because they have not sufficient control of the abdominal muscles in that position. Much more is this the case in the expulsion of the child ... but the recumbent position is sanctioned by custom; it is pointed out as apparently convenient, ... it is dictated by modern laws of obstetrics ... we have all been taught their correctness, and we all thoughtlessly follow their dictates. There is no reason for assuming this (recumbent) position, though we are taught it; it is not reason, or obstetric science, but obstetric fashion which guides us, - guides us through our patients; and blindly do we, like all fashion's votaries, follow in the wake.'

\section{On the upright posture during labour among primitive peoples:}

'I found an extensive literature relating to the subject of posture in labor, but it turns entirely upon the discussion of the relative merits of the dorsal decubitus, as practiced upon the continent of Europe and in America, and the left lateral position, which is favoured in England ... I entered upon the study of the posture occupied by the women of other people ... (and) it soon became evident ... that the recumbent position in labor is rarely assumed among those people who live naturally and are, as yet, governed by their instincts and have escaped the influence of civilization and modern obstetrics. It certainly appeared as if the ordinary obstetrical position of today must be an unnatural one... A vast and important fund of knowledge may be derived from a study of the various positions occupied by women of different peoples in their labors. According to their build, to the shape of their pelvis, they stand, squat, kneel or lie on their belly; so also they vary their position in various stages of labor according to the position of the child's head in the pelvis... The care with which the parturient women of uncivilised people avoid the dorsal decubitus, the modern obstetric position, at the termination of labor, is sufficient evidence that it is a most undesirable position for ordinary cases of confinement; and I am convinced that the thinking obstetrician will soon confirm the statement ... that the recumbent position retards labor and is inimical to easy, safe and rapid delivery... I deem it a great mistake that we in this age of culture, should follow custom or fashion so completely, to the exclusion of reason and instinct, in a mechanical act which so nearly concerns our animal nature as the delivery of the pregnant female... In this purely animal function, instinct will guide the woman more correctly than the varying customs of the times.'

\section{Red Indian management of the third stage of labour:}

'Among our Indians ... massage and expression are freely and effectively used. The third stage of labour is a very short one, the placenta usually escaping very soon after the birth of the child... The patient and her assistants retain the positions relatively occupied during the birth of the child; the parturient, as is commonly the case, kneeling, whilst an assistant, who kneels or stands behind her, with her arms around her waist and the palms of her hands upon the fundus of the uterus, keeps up a steady pressure upon the contracting organ, and in case that the muscular action is not sufficient for the expulsion of the after-birth, she hastens its progress by an effective kneading...

Traction upon the umbilical cord appears so natural, and is certainly so tempting a means of removing the placenta, that it is much esteemed by a class of meddlesome midwives which abounds in all civilized countries to the detriment of parturient women, but the untutored savage, guided in his practice by instinct and observation, is too shrewd to seek the removal of the retained placenta by such dangerous means...

As a rule, we find that the cord is not severed until labor is completed and the secundines are expelled ... the child remaining on the ground in front of the mother until the placenta is delivered...

In regard to the method of cutting the funis, a dull instrument being frequently used ... the cord is also left very long and slowly dries...

In consequence of this method of treatment, the placenta, as a rule, is rapidly expelled, and its retention a matter of very rare occurrence.'

Engelmann, who died in 1903, aged 56, summed up his observations as follows:

'... the obstetric practices of primitive peoples, past and present, are (similar) to our own, and yet although crude, how far in advance in many points - in all such in which simpler means, the hands and external manipulations will answer. The womb they never enter, instruments they have none, but as far as general treatment and external manipulations will reach their management is wonderful, and we will find much to study, to imitate, and to develop.'

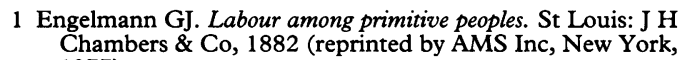
1977). 